\title{
L'interpretazione dei testi matematici tra processi cooperativi e modelli logici: il caso dei connettivi
}

\section{The interpretation of mathematical texts between cooperative processes and logical models: the case of connectives}

\author{
Pier Luigi Ferrari \\ Università del Piemonte Orientale - Italia \\ 凶pierluigi.ferrari@uniupo.it
}

Sunto / Il tema delle competenze logiche richieste per capire la matematica è stato quasi sempre oggetto di controversie sul piano educativo, tra i sostenitori dell'insegnamento della logica come disciplina e quelli che la guardano come una competenza trasversale. Il problema viene spesso sollevato nel corso della scuola secondaria di secondo grado e all'inizio dei corsi universitari. In questo contributo si discutono i processi di interpretazione dei testi matematici in linguaggio verbale, e il potenziale conflitto tra i meccanismi interpretativi propri delle notazioni simboliche della matematica e quelli usuali delle lingue. Viene affrontato in particolare il tema dell'interpretazione dei condizionali, anche attraverso l'esame di due teorie opposte. Vengono poi illustrati alcuni esempi a proposito di altri connettivi proposizionali. La conclusione è che la diversità dei processi interpretativi tra lingua e linguaggi della logica sconsiglia di proporre attività che richiedono l'interpretazione logica di testi verbali al di fuori dei contesti in cui questa sia giustificata.

Parole chiave: lingua; logica; principi di cooperazione; connettivi; bicondizionale.

\begin{abstract}
The subject of the logical competency required to understand mathematics has almost always been the subject of educational controversies, between supporters of teaching logic as a subject and those who look at it as a transversal competency. The problem is often raised in upper secondary school and at the beginning of university courses. This paper discusses the processes of interpretation of mathematical texts in language, and the potential conflict between the mechanisms of interpretation of the symbolic notations of mathematics and the usual ones of languages. In particular, the topic of the interpretation of conditionals is addressed, also through the examination of two opposite theories. Some examples are then illustrated about other propositional connectives. The conclusion is that the diversity of interpretative processes between language and languages of logic does not recommend proposing activities that require the logical interpretation of verbal texts outside the contexts in which this is justified.
\end{abstract}

Keywords: language; logic; cooperative principles; connectives; biconditional.

(C) 2021 Ferrari Pier Luigi. Questo è un articolo Open Access, sottoposto a un processo di revisione tra pari a doppio cieco, pubblicato dal Centro competenze didattica della matematica e dal Servizio comunicazione del DFA-SUPSI in collaborazione con il DECS. L'articolo è distribuito sotto i termini della Licenza Creative Commons Attribuzione 4.0 Internazionale (CC BY 4.0) che permette di usare, condividere e modificare l'articolo su qualsiasi mezzo a patto che l'autore e la fonte originale siano citati. 
Il tema del cosiddetto «pensiero logico» e dei suoi rapporti con l'apprendimento della matematica è stato a lungo, ed è ancora, molto controverso e ha originato impostazioni didattiche e linee di ricerca molto distanti fra loro. Questo può dipendere dalla polisemia della parola «logica», che nella storia del pensiero occidentale ha avuto svariate interpretazioni, da Aristotele ai logici matematici del Novecento, passando per Port-Royal e Hegel. Questo mette in gioco anche i complessi rapporti fra la matematica e la sua didattica, che sono oggetto di diverse interpretazioni che spaziano fra due posizioni estreme: I'approccio pedagogico o psicologico (ma anche filosofico o giuridico ...) che tiene poco conto delle caratteristiche del dominio matematico, e l'identificazione della didattica della matematica con la divulgazione di temi matematici ritenuti intriganti, tenendo poco conto dei processi e delle difficoltà di comunicazione e di apprendimento che intervengono.

Questo tema si ripropone anche in relazione alla pratica di preparare e somministrare prove di vario genere durante gli ultimi anni della secondaria di secondo grado ${ }^{1}$ o all'inizio dell'università (ammissione a determinati corsi, valutazione delle competenze iniziali, ...). Queste prove talvolta sono poco correlate alle Indicazioni Nazionali o alle Linee Guida. In ogni caso, I'esigenza di non dipendere troppo dai curricula della scuola secondaria spinge verso la formulazione di quesiti di vario tipo etichettati come "logici" e anche verso la formulazione di problemi di logica nel linguaggio verbale.

Uno dei punti critici sono i legami tra lo sviluppo del pensiero logico e la logica matematica come disciplina: secondo alcuni I'apprendimento di qualche elemento di logica matematica è un passaggio fondamentale o comunque molto utile per lo sviluppo del pensiero logico. Altri invece contestano questa posizione, e anche le Indicazioni Nazionali per il primo e il secondo ciclo non sembrano suggerire l'insegnamento di elementi di logica matematica. Questa diversità di posizioni è ben illustrata dal titolo di un contributo di Freudenthal (1988) a un convegno sull'insegnamento della logica, tenutosi a Roma nel secolo scorso: Logic as a subject or as an attitude? (La logica come disciplina o come atteggiamento?). ${ }^{2}$ Freudenthal, coerentemente con la sua impostazione realistica, si è schierato nettamente per la seconda opzione. Più di recente è aumentato il numero di chi considera la logica come un tema trasversale. Questo non ha eliminato del tutto le differenze tra le diverse sensibilità. Un punto critico è il funzionamento dei linguaggi che si adottano. Secondo taluni le lingue sono "ambigue", e quindi le notazioni simboliche della logica (o l'uso delle parole corrispondenti in modo conforme alle definizioni logiche) sarebbero più adatte per costruire nuovi enunciati in modo non ambiguo. Questa opinione ha spesso come conseguenza l'introduzione, più o meno formalizzata, di alcune notazioni della logica simbolica, in particolare dei connettivi e delle loro definizioni vero-funzionali, cioè basate sulle tavole di verità. In questo modo pezzetti di logica "come disciplina" rientrano dalla finestra. In questo contributo cercherò di far vedere che è improprio etichettare le lingue come ambigue, mettendo in luce che hanno funzioni diverse dalle notazioni simboliche, o comunque dai linguaggi specifici della logica, funzioni che realizzano, ovviamente, con strumenti diversi. Saranno discusse in via preliminare le Indicazioni Nazionali per il primo e il secondo ciclo per quanto riguarda i temi collegati alla logica. Infine, saranno discussi i diversi usi di alcuni termini che in logica vengono chiamati connettivi e nelle lingue possono appartenere a categorie grammaticali diverse.

1. La scuola secondaria di secondo grado in Italia dura cinque anni e corrisponde all'ultimo anno di scuola media e alla scuola media superiore o scuole professionali nel Canton Ticino.

2. Freudenthal usava la parola «attitude» («atteggiamento») in un senso più ampio rispetto a quello specifico prevalente nell'educazione matematica odierna. 


\section{Le Indicazioni Nazionali}

Nelle Indicazioni Nazionali non vi è nessun riferimento all'insegnamento della logica come disciplina. Può essere interessante andare a vedere come viene usato l'aggettivo logico nelle sue declinazioni di genere e numero.

Nelle Indicazioni Nazionali per il primo ciclo l'aggettivo logico compare 11 volte, in un caso al plurale. 8 occorrenze sono collocate in paragrafi che riguardano l'apprendimento linguistico, spesso in combinazione con altri aggettivi: "pensiero logico e creativo»; "strutturazione logico-concettuale»; «organizzazione logico-sintattica» (4 volte); «ordine cronologico e logico» (2 volte). Una occorrenza («pensiero logico e spaziale») è una citazione a piè di pagina delle competenze-chiave elencate nella Raccomandazione del Parlamento europeo e del Consiglio del 18 dicembre 2006 e un'altra («Dimostra prime abilità di tipo logico») riguarda le competenze di base attese alla fine della scuola dell'infanzia. Infine, nei Traguardi per lo sviluppo delle competenze al termine della scuola primaria per la matematica, si trova «Legge e comprende testi che coinvolgono aspetti logici e matematici». Nelle Indicazioni Nazionali per il secondo ciclo, se ci limitiamo a un solo percorso liceale, I'aggettivo logico, nelle sue varie declinazioni, compare 11 volte, 2 delle quali nella nota introduttiva (area «logico-argomentativa», «ragionare con rigore logico»), 3 tra le linee generali e competenze o gli obiettivi specifici di apprendimento di filosofia («problemi logici», «logica moderna», «sviluppi della logica»), 4 tra le linee generali e competenze di lingua e letteratura italiana (competenze «logico-argomentative», «coerenza logico-argomentativa», "organizzazione logica», «relazioni logiche interne») e 2 nelle linee generali di scienze («connessioni logiche» e un riferimento alla logica non lineare delle tappe di un percorso di apprendimento).

È quindi evidente che la dimensione logica viene proposta come trasversale e legata in primo luogo all'educazione linguistica, ma anche alla filosofia, alle scienze e alla matematica. È anche rilevante che nel secondo ciclo venga considerata un'area «logico-argomentativa» autonoma rispetto a quella «linguistica e comunicativa», a quella «storico-umanistica» e a quella "scientifica, matematica e tecnologica».

\section{Le lingue sono ambigue?}

Come accennato nell'introduzione, il tema dei rapporti tra sviluppo del pensiero logico e insegnamento di elementi di logica matematica si ripropone anche in relazione alle prove di vario genere somministrate all'inizio dell'università. In queste prove spesso problemi di logica sono formulati in linguaggio verbale. In questi ultimi casi il problema dell'interpretazione dei testi verbali di natura logica si pone apertamente, dato che i meccanismi interpretativi delle lingue e dei linguaggi simbolici della logica sono diversi. In logica matematica è fondamentale poter applicare algoritmi alle espressioni. Per i linguaggi simbolici, come i linguaggi del primo ordine, quelli più ampiamente usati in logica, vale il teorema di leggibilità unica, che stabilisce che data una sequenza finita di simboli è possibile determinare un modo univoco se la sequenza è una formula, qual è la sua struttura e quali sono le sue sottoformule. ${ }^{3}$ Senza un teorema di unicità di lettura sarebbe problematico svolgere le numerose dimostrazioni per induzione di proprietà dei linguaggi del primo ordine.

Le lingue non dispongono di un teorema di leggibilità unica ma hanno altri strumenti potenti per

3. Si veda ad esempio Lolli (1991, p. 35). 
disambiguare i testi quando è necessario, e per non renderli inutilmente prolissi quando non serve. Bisogna rendersi conto che le lingue hanno sì la funzione di trasmettere informazioni, ma anche di intrattenere rapporti con gli altri, esprimere le proprie emozioni, dare ordini, mettere in atto decisioni. Inoltre, le lingue costruiscono i significati non solo attraverso il lessico e i connettivi vero-funzionali ma anche in altri modi, come ad esempio l'organizzazione del testo o anche strumenti non testuali (come il tono della voce o l'espressione del viso). Riguardo al primo caso, due enunciati come «Quel ristorante è caro ma buono»

e

«Quel ristorante è buono ma caro»

sono equivalenti dal punto di vista logico (entrambi esprimono la congiunzione logica degli stessi enunciati) ma comunicano messaggi diversi.

Considerare le caratteristiche delle lingue significa tener conto del fatto che sono configurate per operare in contesti diversi e per realizzare funzioni diverse, fra le quali anche quelle logiche. Questo mette inevitabilmente in gioco la loro dimensione pragmatica. I temi classici della pragmatica, che, forse non casualmente, sono tutti collegati a questioni studiate in educazione matematica, sono gli indicali, gli atti linguistici e la cooperazione comunicativa.

Gli indicali sono parole la cui interpretazione richiede informazioni sia sul contesto costituito dal testo sia da quello extralinguistico. II contesto extralinguistico comprende il tempo in cui il testo viene prodotto (oggi, domani, l'anno scorso...), gli interlocutori (io, tu, noi...) e la collocazione spaziale (questo, quello, laggiù, qui). In altri casi il riferimento è a una parte precedente del testo. Nella frase «Siamo stati a Venezia. La città è attraversata da numerosi canali», I'espressione «La città» è riferita a Venezia, e l'enunciato "La città è attraversata da numerosi canali» è vero. Se la frase fosse «Siamo stati ad Asti. La città è attraversata da numerosi canali», il riferimento di "La città» sarebbe Asti, e I'enunciato «La città è attraversata da numerosi canali» sarebbe falso. Per approfondimenti su questi aspetti, e anche sulla pragmatica in generale, si veda il lavoro di Bazzanella (2008).

Un atto linguistico è legato al fatto di produrre un enunciato in un determinato contesto. Esso riguarda anche le frasi non dichiarative (interiezioni, ordini, domande, ...) e comprende il fatto di produrre quell'enunciato in quelle circostanze; può quindi esprimere atteggiamenti, convinzioni, impegni, azioni del parlante o modificare atteggiamenti, convinzioni, comportamenti del ricevente. Una proposizione è quindi quella componente del significato di un enunciato che consente di identificare i referenti e stabilire se l'enunciato è vero o falso. Ad esempio, dire «Piove» a qualcuno che sta uscendo non comunica solo un'informazione (vera o falsa che sia) ma può essere un invito a prendere I'ombrello. Un atto linguistico non è caratterizzato soltanto dalla sua verità o falsità, ma anche dalla sua adeguatezza rispetto al contesto. Dire «Piove» a qualcuno che è appena entrato in casa bagnato fradicio potrebbe essere un atto linguistico inadeguato, in quanto comunicherebbe un'informazione inutile, ancorché vera. La teoria degli atti linguistici è dovuta ad Austin (1962). Per approfondimenti si veda anche la raccolta curata da Sbisà (1978).

La cooperazione comunicativa è un fenomeno messo in luce da Grice (1975), che ha formulato il Principio di Cooperazione. Secondo tale principio ogni partecipante a uno scambio conforma il proprio contributo a quanto è richiesto, nel momento in cui avviene, dall'intento comune accettato o dalla direzione dello scambio verbale in cui è impegnato. II principio si articola in quattro massime: della quantità («Dai un contributo appropriato sotto il profilo della quantità di informazioni»); della qualità («Non dire cose che credi false o che non hai ragione di credere vere»); della relazione («Dai un contributo pertinente a ogni stadio della comunicazione»); del modo («Esprimiti in modo chiaro, breve, ordinato»). II principio di Grice è un principio descrittivo, che spiega come i partecipanti si comportano durante un dialogo e può essere applicato in modi diversi, in base agli scopi. Essendo una descrizione di ciò che accade può essere anche disatteso, violato o persino contraddetto. La violazione del principio produce in ogni caso effetti comunicativi.

Quando un enunciato è prodotto o ricevuto in un contesto (cioè, sempre) i fenomeni pragmatici si 
innescano inevitabilmente, che piaccia o no. Essi non sono da considerarsi deviazioni o sgradite eccezioni, perché sono il motore stesso della comunicazione: senza di essi sarebbe impossibile comunicare. Il principio fondamentale che regola gli scambi fra persone non è quindi la verità degli enunciati in gioco ma la loro adeguatezza rispetto a un contesto. Nessuna persona assennata si sognerebbe di telefonare a qualcuno alle tre del mattino per dirgli che Parigi è la capitale della Francia. La proposizione associata sarebbe certamente vera, ma l'atto linguistico sarebbe del tutto inadeguato. Se io ricevessi una telefonata a quell'ora mi preoccuperei molto, perché, assumendo (come si fa sempre, con rare eccezioni) che chi chiama (chiunque sia) rispetti il principio di cooperazione, dovrei aspettarmi una notizia cattiva e grave. L'alternativa è che la telefonata sia dovuta a un errore, nel qual caso la violazione del principio di cooperazione sarebbe non intenzionale e quindi non informativa.

Va comunque detto che i fenomeni pragmatici avvengono con diversi livelli di consapevolezza dei partecipanti. Ad esempio, I'applicazione o la violazione del Principio di Cooperazione non sono sempre consapevoli. Gombert (1990) ha studiato diversi aspetti della consapevolezza nell'uso della lingua, come ad esempio le reazioni di bambini dai 4 ai 6 anni esposti a enunciati non cooperativi prodotti da adulti.

Nei testi di vario tipo che trattano questi temi il linguaggio della logica viene interpretato, più o meno consapevolmente, come un modello di linguaggio rigoroso. Le lingue vengono invece etichettate come ambigue. Talvolta la presunta ambiguità delle lingue viene motivata con esempi di frasi la cui struttura sintattica non è univoca, come

«Una vecchia porta la sbarra» (1)

in cui alcune parole possono appartenere a categorie grammaticali diverse: «vecchia» può essere nome o aggettivo, «porta» verbo o nome, «la» articolo o pronome, «sbarra» nome o verbo. Altre volte vengono proposti esempi in cui la struttura sintattica è univoca ma alcune parole hanno più significati, come

«ll delfino nuotò allegramente in piscina il giorno in cui suo padre perse la testa» (2)

Esempi di questo tipo non sono a mio giudizio particolarmente significativi. Bisogna prendere atto che le lingue dispongono di strumenti molto potenti per disambiguare le espressioni, sia in fase di produzione, sia in fase di ricezione.

Una frase come la (1), che può essere letta in due modi grammaticalmente corretti, difficilmente potrebbe generare confusione, nei contesti in cui sono normalmente usate le lingue. Un emittente cooperativo avrebbe a disposizione svariate possibilità per rendere la frase univoca, e se non le sfruttasse violerebbe il principio di cooperazione. Ad esempio, con «Una vecchietta porta la sbarra» potrebbe suggerire una delle due interpretazioni e con «Una porta vecchia la sbarra» I'altra. Nel parlato, la frase sarebbe pronunciata con diverso posizionamento delle pause. Allo stesso modo un ricevente potrebbe interpretare la frase basandosi sul contesto, che dovrebbe comunque essere definito per consentire l'interpretazione di «la», articolo o pronome che sia. Allo stesso modo, una «D» maiuscola nel caso di testo scritto sarebbe sufficiente per disambiguare la (2), e in ogni caso la netta differenza di contesti renderebbe molto improbabili gli equivoci. Occorre accettare l'idea che le lingue vengono usate comunemente e quasi sempre felicemente per comunicare e che funzionano in modi diversi rispetto ai sistemi simbolici della matematica, per raggiungere i loro scopi, che sono diversi. La presunta ambiguità delle lingue deriva in parte dal fatto che non godono delle stesse proprietà di decidibilità dei linguaggi simbolici della logica, in parte dalle difficoltà di conversione da linguaggio verbale a linguaggi logici. Quindi chi sostiene che le lingue sono ambigue probabilmente sta applicando a esse i criteri usati per i sistemi simbolici. Se, simmetricamente, applicassimo a questi ultimi i criteri di funzionamento delle lingue, dovremmo dire che violano platealmente i principi cooperativi. $V a$ inoltre aggiunto che un qualche contesto è comunque necessario per ricostruire il significato tanto dei testi verbali come delle espressioni simboliche anche quando siano leggibili in modo univoco. Una frase come «Ho lasciato la macchina dal gommista» è grammaticalmente univoca, ma solo attraverso inferenze basate sul contesto è possibile determinarne il significato. La frase non dice di quale 
macchina si tratta (automobile, o macchina per cucire, o macchina di Turing?) né di quale gommista. Analogamente, per sapere se una formula come

$$
x^{2}+1=0
$$

è soddisfacibile avremmo bisogno di conoscere il dominio in cui può variare la $x$.

Tornando al tema di questo paragrafo, è quindi opportuno abbandonare l'idea che il linguaggio quotidiano sia costituito da un'ossatura logica rigorosa, con l'aggiunta di ambiguità, imprecisioni ed eccezioni, magari attribuiti a ragioni psicologiche o storiche, che ne danneggiano la correttezza o I'eleganza. Se vogliamo andare oltre gli stereotipi e cominciare a capire il ruolo delle lingue in educazione matematica, dobbiamo adottare la prospettiva opposta: nella quasi totalità delle situazioni, compresa la maggior parte di quelle in cui si fa matematica, le lingue funzionano sulla base dei principi pragmatici. ${ }^{4}$ In alcuni casi vengono utilizzati linguaggi specifici, come ad esempio le notazioni matematiche, con funzionamenti e scopi diversi, come ad esempio il calcolo, numerico o simbolico. Anche le lingue possono essere usate adottando alcune caratteristiche dei linguaggi simbolici, ma i fenomeni pragmatici di cui sopra rimangono comunque attivi.

Un confronto più approfondito fra le lingue e i linguaggi della matematica è stato sviluppato da Ferrari (2021).

\section{I condizionali}

\subsection{L'ipotesi del bicondizionale}

La riflessione riguardo alle influenze del linguaggio ordinario e dei suoi schemi interpretativi sulla risoluzione di problemi di logica è stata stimolata dai tentativi di spiegare alcuni errori nell'uso delle costruzioni condizionali.

Vediamo un esempio, preso da Rumain et al. (1983), del tipo «Negazione dell'antecedente» (NA). Se $P$ è l'enunciato

«Nella scatola c'è un cane»

e Q è

«Nella scatola c'è una mela»,

da «Se $P$ allora $Q$ » e «Non P» numerosi soggetti, bambini e adulti, ricavavano la conclusione logicamente sbagliata «Non $Q »$.

Analogamente, nei problemi del tipo «Affermazione del conseguente» $(A C)$, da «Se $P$ allora $Q$ » e «Q», un numero analogo di soggetti ricavava la conclusione sbagliata «P».

La spiegazione dominante era la cosiddetta teoria del bicondizionale (Matalon, 1962; Peel, 1967; Taplin et al., 1974) secondo la quale l'errore stava nel fatto che il condizionale veniva interpretato come se fosse un bicondizionale (cioè, «P se e solo se $Q »)$ ). L'errore era quindi collocato nel piano logico e non si riteneva che la difficoltà fosse collegata anche all'interpretazione del testo. Una diversa, e più convincente, spiegazione è stata offerta da Rumain et al. (1983), secondo i quali i processi di comprensione colloquiali sono all'origine dei comportamenti rilevati. In altre parole, un enunciato come

«Se nella scatola c'è un cane allora nella scatola c'è una mela»

suggerirebbe l'inferenza

«Se nella scatola non c'è un cane allora nella scatola non c'è una mela».

Le inferenze suggerite possono essere chiamate, con quello che allora era considerato un neologi-

4. Non mi riferisco al solo principio di cooperazione di Grice ma anche ad altri, come il principio di rilevanza di Sperber e Wilson (1986). 
smo, ${ }^{5}$ implicature conversazionali. Un'implicatura conversazionale è quella parte di informazione ricavabile da un testo che non deriva dal suo contenuto dichiarativo ma piuttosto dall'ipotesi che il testo sia adeguato al contesto. II fatto che sia citata la presenza del cane nella scatola come condizione per la presenza della mela porta a ritenere che tale condizione sia rilevante (come avviene usualmente nella comunicazione fra persone) e che in sua assenza venga meno anche la conclusione. Nei loro esperimenti gli autori utilizzano diversi accorgimenti per disinnescare le inferenze suggerite, ad esempio aggiungendo premesse maggiori. Se si assumono le tre premesse:

1. "Se nella scatola c'è un cane allora nella scatola c'è una mela»

2. "Se nella scatola c'è un gatto allora nella scatola c'è una pera»

3. «Se nella scatola c'è un coniglio allora nella scatola c'è una mela»

l'informazione

«Nella scatola non c'è un cane»

non porterebbe più così frequentemente alla conclusione

«Nella scatola non c'è una mela»

in quanto la premessa 3 disinnescherebbe il suggerimento implicito nella condizione «Nella scatola c'è un cane».

È evidente che secondo l'ipotesi del bicondizionale l'aggiunta di premesse non dovrebbe provocare variazioni nelle risposte. I risultati sperimentali hanno confermato le ipotesi. L'interesse dello studio di Rumain et al. (1983), comunque, sta più nell'idea di chiamare in causa il linguaggio che nell'apparato sperimentale o nel quadro di riferimento: i condizionali esaminati rappresentano comunque relazioni arbitrarie fra enunciati, mentre nella vita quotidiana i condizionali si usano prevalentemente per esprimere connessioni semantiche. Questo aspetto è stato studiato anche attraverso il famoso test di Wason, ${ }^{6}$ in cui si è richiesto ad alcuni soggetti di applicare una regola arbitraria, come nella versione che segue.

Viene mostrata una serie di quattro carte posizionate su un tavolo, ognuna delle quali ha un numero su una faccia e un colore sull'altra. Le facce visibili delle carte mostrano «3», «8», «rosso» e «marrone». Si richiede al soggetto di stabilire quale carta o carte deve girare per verificare la verità della seguente proposizione: se una carta mostra un numero pari su una faccia, allora la sua faccia opposta è rossa. Meno del $10 \%$ dei soggetti ha dato la risposta appropriata, e cioè che bisogna girare la carta con 8 e quella con la faccia marrone. Successivi esperimenti hanno messo in luce come problemi logicamente equivalenti ma immersi in un contesto in cui la regola diventava significativa e socialmente accettata ricevevano un numero di risposte corrette molto superiore. L'esperimento di Wason è stato ampiamente discusso soprattutto in ambito psicologico. A noi serve essenzialmente a confermare che l'arbitrarietà dei legami tra antecedente e conseguente è un fattore che influenza pesantemente I'interpretazione dei condizionali. Questo non dipende soltanto dalle situazioni in cui sono collocati gli esperimenti e la loro maggiore o minore familiarità, ma anche dalla formulazione dei testi. L'esempio che segue può aiutare a chiarire le idee.

Un esame consiste nel sostenere due prove a quiz. II punteggio di ogni quiz è un numero intero da 0 a 10. Si supera l'esame se e solo se la somma dei due punteggi è non inferiore a 12.

\section{Caso 1}

Lo studente A guarda il risultato della prima prova e dice "Se nella seconda prendo 8 ho superato l'esame». Sappiamo che nella prima prova ha preso 4. L'affermazione di A è vera? La risposta è sì. Su questo normalmente c'è ampio consenso.

5. Il lavoro di Rumain et al. è successivo di soli otto anni all'edizione inglese del lavoro di Grice (1975) in cui viene discussa l'idea di implicatura conversazionale.

6. Si vedano Johnson-Laird (1983) e Cosmides e Tooby (1992) per panoramiche sul test e alcune ricerche successive, o anche Houdé et al. (1992) per un'analisi dal punto di vista delle neuroscienze. 


\section{Caso 2}

Lo studente A guarda il risultato della prima prova e dice «Se nella seconda prendo 8 ho superato I'esame». Sappiamo che nella prima prova ha preso 6. L'affermazione di A è vera?

La risposta è ancora sì, anche se qui si viola il principio di cooperazione. Qui diversi studenti sarebbero indotti a rispondere che non è vera, in quanto l'affermazione di A contiene un elemento arbitrario (perché proprio 8?). La condizione contenuta nell'antecedente è solo sufficiente, ma questo accadeva anche nel caso 1. La differenza è che nel caso 1 la scelta di " 8 " non era arbitraria, essendo il minimo voto utile, mentre nel caso 2 si verifica il contrario.

\section{Caso 3}

Lo studente A guarda il risultato della prima prova, che è 6 , e chiede al professore se ha già corretto la seconda prova. II professore dice: «Sì, I'ho corretta. Non ricordo bene ma mi sembra che il tuo voto nella seconda prova sia 8». Lo studente A replica: «Se nella seconda ho preso 8 ho superato l'esame». L'affermazione di A è vera?

La risposta è ancora sì, e qui normalmente gli studenti non hanno esitazioni. Infatti, il principio di cooperazione non è violato in quanto l'enunciato del professore, anche se non dà alcun contributo informativo affidabile, rende tuttavia rilevante l'enunciato finale di A, eliminando I'arbitrarietà che di fatto violava la massima della relazione.

\subsection{Il condizionale nella lingua}

In logica il condizionale "Se $P$ allora $Q$ (chiamato in ambito filosofico implicazione materiale) è un enunciato che è vero in tutti i casi in cui $P$ è falso oppure $Q$ è vero. Questo vale anche in assenza di legami semantici fra $P$ e $Q$.

Nella maggior parte delle lingue il condizionale svolge funzioni diverse e più complesse. In primo luogo, nelle lingue esistono modi e tempi, quindi può accadere che gli enunciati messi in relazione avvengano in tempi diversi, o con gradi di certezza diversi, originando asimmetrie che in logica non ci sono. In secondo luogo, nelle lingue non ci sono solo proposizioni che possono essere vere o false ma, come visto nel par. 3, atti linguistici.

Vediamo qualche esempio. Nel caso più semplice abbiamo un evento che ne causa o influenza un altro, come nell'enunciato

«Se piove allora Mario ha l'ombrello aperto».

In questo caso abbiamo un legame tra due fatti, la pioggia induce Mario ad aprire l'ombrello.

Invece il condizionale

«Se Mario ha l'ombrello aperto allora piove»

non è l'inverso del precedente. Qui il parlante non intende affermare che l'apertura dell'ombrello di Mario provoca la pioggia ma che da quella è possibile ricavare l'informazione che piove. Qui il legame è tra un fatto e la conoscenza di un altro fatto (condizionale epistemico).

Un altro gruppo di condizionali si ha quando la condizione riguarda non il contenuto proposizionale ma l'atto linguistico, come nel classico esempio

"Se hai fame c'è della torta in frigo».

In questo caso è evidente che la torta in frigo c'è o non c'è indipendentemente dalla fame dell'interlocutore. La condizione «Se hai fame» non ha a che fare con la verità del conseguente («c'è della torta in frigo») ma con l'adeguatezza dell'atto linguistico. La semplice affermazione "C'è della torta in frigo» potrebbe apparire irrilevante, specie se l'interlocutore non avesse fame. Quindi il "Se hai fame» potrebbe essere parafrasato da "Attenzione: quello che sto per dirti ha senso se hai fame». Allo stesso modo un condizionale può essere usato in risposta a una richiesta non chiara. A uno studente che sapeva di non essere stato ammesso all'orale ma insisteva per conoscere «l'esito» del suo scritto mi è capitato di rispondere qualcosa come "se per "esito" intende il voto, allora è 12». 
In questo caso il voto è comunque 12 , I'antecedente del condizionale serve solamente a delimitare il dominio di adeguatezza del conseguente.

\section{Altri connettivi}

\subsection{La negazione}

Fenomeni simili avvengono con la negazione. Spesso i confronti fra le lingue e le notazioni della logica si focalizzano sul problema della doppia negazione. Tuttavia, è difficile sviluppare seriamente paragoni tra una notazione che dispone esclusivamente di un operatore unario di negazione e una lingua che dispone di svariati modi per negare un enunciato, o per graduarne verità, affidabilità o adeguatezza secondo una scala di valori ricca di sfumature e che va ben al di là della scelta fra vero e falso. D'altra parte, anche nei linguaggi del primo ordine due negazioni non sempre si annullano. Se usiamo « $\neg$ 》 come operatore unario di negazione e «P» è un enunciato, allora «P» e « $\neg P$ » sono equivalenti, almeno in logica classica. Ma se le occorrenze della negazione non sono consecutive, I'equivalenza potrebbe saltare. Ad esempio l'enunciato

$\|_{\neg} \forall x_{\neg} P(x) »$ («Non per tutti gli $x$ vale non $\left.P(x) »\right)(1)$

in generale non è equivalente $a$

« $\forall x P(x) »$ («Per tutti gli $x$ vale $P(x) »)$. (2)

Quindi la regola (o proverbio?) «due negazioni affermano» va presa con le molle anche in logica matematica.

Nelle lingue la negazione non è sempre vero-funzionale, cioè non sempre trasforma un enunciato in un altro il cui valore di verità è opposto (qualunque cosa voglia dire "opposto").

Due enunciati come

«Questo quadro è bello» (3)

e

«Questo quadro non è bello, è orribile» (4)

esprimono sostanzialmente giudizi opposti o, quanto meno, incompatibili. Invece l'enunciato

"Questo quadro non è bello, è un capolavoro» (5)

non esprime un giudizio opposto rispetto a (3) e non intendere mettere in dubbio la bellezza del quadro. Quello che viene messo in dubbio è l'adeguatezza dell'atto linguistico collegato a (3).

In qualche altro caso la negazione può mettere in discussione la forma dell'enunciato, come in

«Tu non vai a squola, vai a scuola» (6),

dove viene messa in discussione la grafia di una parola, oppure

«Mario non è il padre di Roberta, è Roberta la figlia di Mario» (7)

in cui viene messa in discussione l'organizzazione testuale di un qualche precedente enunciato. In questo caso, per chi conosce Mario ma non Roberta è più informativo pensare a Roberta come alla figlia di Mario, mentre per chi conosce Roberta ma non Mario avrebbe più senso la scelta simmetrica. Nel linguaggio verbale, quindi, la negazione è caratterizzata da una complessità molto maggiore (sono svariati i modi di negare, lessicali o no) e anche dal fatto che gli enunciati sono inseriti, piaccia o non piaccia, in atti linguistici, e negare gli uni o gli altri non è sempre equivalente.

\subsection{La congiunzione logica}

La congiunzione logica è associata usualmente alla congiunzione «e», che però in italiano ha svariati usi. Nel caso di predicati di due o più argomenti la «e» può giocare il ruolo di separatore fra questi ultimi, come nel caso 
«7 è la media di 5 e 9» (8)

che ovviamente non è la congiunzione logica di «7 è la media di 5 » e «7 è la media di 9». Nella stessa categoria rientrano anche gli esempi, tristemente diffusi nei testi ai tempi della "matematica moderna", come

«Ho un maglione giallo e rosso» (9)

che ovviamente non è la congiunzione logica di

«Ho un maglione giallo» (10)

e

«Ho un maglione rosso». (11)

Talvolta la «e» può prendere un significato molto simile alla «o» come nel caso:

«Vino e grappa mi provocano mal di stomaco» (12),

dove probabilmente si fa riferimento all'assunzione di una cosa qualunque fra vino e grappa, e non a quella di una bevanda che li contenga entrambi in associazione. Altre volte la «e» può essere utilizzata per costruire forme assimilabili a dei condizionali, come in

«Studia e sarai promosso» (13).

Ci si è spesso soffermati sul fatto che in moltissimi casi nelle lingue la «e» non è commutativa, come accade invece in logica. Un esempio classico è la coppia

«Ho cambiato strada e ho visto Gianni» (14)

e

«Ho visto Gianni e ho cambiato strada» (15)

in cui l'inversione dell'ordine provoca una drastica variazione nel significato. Questo può dipendere dal fattore tempo (sia quello degli eventi narrati, sia i tempi connessi alle azioni di scrivere, pronunciare, leggere o ascoltare un testo) che in logica classica è assente, o anche da altri fattori. In qualche caso viene cambiato non solo il significato dell'enunciato composto ma anche la sua adeguatezza, come nella coppia

«Sono arrivato in stazione e ho preso il treno» (16)

e

«Ho preso il treno e sono arrivato in stazione» (17).

L'enunciato (16) dà informazioni che in alcuni contesti possono essere rilevanti. Non dice qual è la stazione e nemmeno qual è il treno, ma queste informazioni potrebbero essere (ritenute) irrilevanti, $\mathrm{o}$, in caso contrario, il ricevente potrebbe ricavarle dal contesto. Però dice che è andato in stazione (e non è rimasto a casa, o non è andato al mare) e che vi è andato per prendere un treno (e non per prendere un giornale all'edicola o un caffè al bar). Invece la (17), in un'interpretazione standard, è priva di informazioni rilevanti. La prima interpretazione che salta in mente è che l'emittente abbia prima preso un treno e poi sia arrivato in una stazione. In questo caso l'informazione è poco rilevante: è normale per chi prende un treno arrivare in una stazione, la massima della quantità, qui evidentemente violata, richiederebbe di specificare almeno la località di arrivo.

Le differenze tra la congiunzione logica e l'uso di «e» nella lingua dipendono quindi da diversi fattori: la presenza del tempo (come visto sopra) ma anche l'organizzazione testuale. Anche questi fattori sono usati con profitto a scopi comunicativi.

\subsection{La disgiunzione}

L'uso prevalente della "O» inclusiva in matematica è probabilmente motivato dalle stesse ragioni per cui in matematica diversi attributi sono usati inclusivamente. In matematica è corretto, e spesso opportuno, denominare «rettangolo» un quadrato, mentre dal punto di vista della comunicazione quotidiana questo potrebbe risultare poco efficace, in quanto dicendo «rettangolo» molto spesso si intende «non quadrato». Questa differenza non sta nella definizione di «rettangolo» (in nessun dizionario c'è scritto che un rettangolo non può essere quadrato), ma nell'uso della parola. Con la 
disgiunzione inclusiva accadono fenomeni dello stesso tipo. Sono note le difficoltà da parte di diversi studenti ad accettare formule come

«3 $\leq 100 »$,

che, se convertita in linguaggio verbale diventa

«tre è minore o uguale a 100 ».

L'uso inclusivo porta quasi inevitabilmente a violare la massima della quantità: I'aggiunta «o uguale»

aumenta la complessità dell'enunciato e allo stesso tempo ne riduce il grado di informatività. Naturalmente esistono contesti in cui tale uso non viola i principi cooperativi. In genere sono contesti in cui esistono regole precise e motivate. Ad esempio, davanti a un dipartimento universitario, in tempi di Covid-19, era affisso un cartello con il testo seguente:

«Possono accedere al terzo piano solo gli studenti che siano iscritti all'esame di Istologia oppure dotati di autorizzazione di accesso ai laboratori».

In questo caso dal contesto è chiaro che potrebbero esserci studenti in possesso di uno solo dei due requisiti, o anche di entrambi.

Nel caso della disgiunzione entrano pesantemente in gioco i principi cooperativi: ci sono situazioni in cui l'uso della disgiunzione inclusiva in luogo di altre forme li viola.

\section{Conclusioni}

Con questa carrellata di esempi si è cercato di mettere in luce come i meccanismi di interpretazione delle lingue e dei sistemi simbolici della matematica siano diversi, anche se non contrapposti. Ferrari (2021) ha evidenziato che gli usi matematici sono forme estreme di registri colti, cioè delle varietà d'uso della lingua adottate usualmente nella comunicazione scientifica e in molti altri contesti. Vi è quindi continuità tra le due forme di interpretazione, ma anche qualche differenza. Le differenze si basano sulle diverse funzioni dei linguaggi. Per questo la scelta migliore non è quella di presentare le due modalità come contrapposte, magari suggerendo che le lingue siano ambigue o vaghe, mentre le notazioni matematiche sarebbero rigorose e precise. È preferibile invece da un lato prendere atto della diversità di funzioni e magari rifletterci sopra, e dall'altro scegliere come terreno per i primi incontri con la logica quei contesti in cui le interpretazioni logiche e quelle usuali della lingua non siano in netto contrasto.

\section{Bibliografia}

Austin, J. L. (1962). How to do things with words. Harvard U. P.

Bazzanella, C. (2008). Linguistica e pragmatica del linguaggio. Un'introduzione. Laterza.

Cosmides, L., \& Tooby, J. (1992). Evolutionary psychology: A Primer. https://www.cep.ucsb.edu/primer.html

Ferrari, P. L. (2021). Educazione matematica, lingua, linguaggi. Costruire, condividere e comunicare matematica in classe. Utet.

Freudenthal, H. (1988). Logic as a subject or as an attitude? In M. Barra \& A. Zanardo (A cura di), Atti del XII incontro di Logica Matematica. La logica matematica nella didattica (pp. 27-38). 
Gombert, J. É. (1990). Le Développement Métalinguistique. Presses Universitaires de France.

Grice, H. P. (1975). Logic and conversation. In P. Cole \& J. L. Morgan (Eds.), Syntax and semantics: Vol.3. Speech acts (pp. 41-58). Academic Press.

Houdé, O., Zago, L., Mellet, E., Moutier, S., Pineau, A., Mazoyer, B., \& Tzourio-Mazoyer, N. (2000). Shifting from the perceptual brain to the logical brain: the neural impact of cognitive inhibition training. Journal of Cognitive Neuroscience, 12(5), 721-728.

Johnson-Laird, P. N. (1983). Mental Models. Towards a Cognitive Science of Language, Inference and Consciousness. Cambridge University Press.

Lolli, G. (1991). Introduzione alla logica formale. il Mulino.

Matalon, B. (1962). Etude génétique de I'implication. In E. W. Beth et al. (Eds.), Implication, formalisation, et logique naturelle. Presses Universitaires.

Peel, E. A. (1967). A method for investigating children's understanding of certain logical connectives used in binary propositional thinking. British Journal of Mathematical and Statistical Psychology, 20, 81-82.

Rumain, B., Connell, J., \& Braine, M. D. S. (1983). Conversational Comprehension Processes Are Responsible for Reasoning Fallacies in Children As Well As Adults: If Is Not the Biconditional. Developmental Psychology, 19(4), 471-481.

Sbisà, M. (A cura di). (1978). Gli atti linguistici. Feltrinelli.

Sperber, D., \& Wilson, D. (1986). Relevance: communication and cognition. Basil Blackwell.

Taplin, J. E., Staudenmayer, H., \& Taddonio, J. L. (1974). Developmental changes in conditional reasoning: Linguistic or logical? Journal of Experimental Child Psychology, 17, 360-373.

Villani, V., Bernardi, C., Zoccante, S., \& Porcaro, R. (2012). Non solo calcoli. Domande e risposte sui perché della matematica. Springer. 